\title{
Frontières
}

\section{L'utilisation du génogramme dans la clinique du suicide}

\section{Normand d'Aragon}

Volume 12, numéro 1, automne 1999

Suicides, générations et culture

URI : https://id.erudit.org/iderudit/1074512ar

DOI : https://doi.org/10.7202/1074512ar

Aller au sommaire du numéro

Éditeur(s)

Université du Québec à Montréal

ISSN

1180-3479 (imprimé)

1916-0976 (numérique)

Découvrir la revue

Citer cet article

d'Aragon, N. (1999). L’utilisation du génogramme dans la clinique du suicide. Frontières, 12(1), 73-78. https://doi.org/10.7202/1074512ar

\section{Résumé de l'article}

Description de l'utilisation du génogramme comme outil d'évaluation et d'intervention dans la clinique du risque de passage à l'acte suicidaire ou du deuil par suicide. Illustration de la rencontre des facteurs intrapsychiques et interactionnels. L'accent est placé sur l'influence des deuils jamais résolus dans la ou les générations précédentes dans la dynamique familiale. Le modèle d'analyse s'inspire de l'approche familiale intergénérationnelle. d'utilisation que vous pouvez consulter en ligne.

https://apropos.erudit.org/fr/usagers/politique-dutilisation/ 


\section{Résumé}

Description de l'utilisation du génogramme comme outil d'évaluation et d'intervention dans la clinique du risque de passage à l'acte suicidaire ou du deuil par suicide. Illustration de la rencontre des facteurs intrapsychiques et interactionnels. L'accent est placé sur l'influence des deuils jamais résolus dans la ou les générations précédentes dans la dynamique familiale. Le modèle d'analyse s'inspire de l'approche familiale intergénérationnelle.

Mots clés: suicide - famille - génogramme.

\begin{abstract}
Description of the use of the genogram as an in-clinic evaluation and intervention tool for the risk of act of suicide or for grief caused by suicide. Illustration of the convergence of intrapsychic and interactional factors. Emphasis is placed on the influence of unresolved grief within the previous generations in the family dynamic. The analytical model refers to the intergenerational family approach.
\end{abstract}

Key words: suicide - family - genogram

\section{L'utilisation du génogramme dans la clinique du suicide}

Normand d'Aragon,

psychologue au CLSC et CSHLD de la MRC de l'Islet.

\section{UN ENCHAÎNEMENT \\ D'EMPREINTES}

L'intervention clinique auprès des personnes et familles en contexte de risque suicidaire ou de deuil par suicide nous amène à voir dans cette problématique le reflet d'une impasse développementale et interactionnelle ${ }^{1}$, impliquant bien souvent toute la famille. Avec une grille d'analyse appropriée, elle peut être comprise comme un révélateur mais aussi une occasion de "guérir» des séquelles de deuils jamais résolus dans l'histoire de l'une ou des deux lignées d'origine ${ }^{2}$.

L'incapacité de résoudre un deuil familial en une génération aura bloqué certains aspects du développement de la personnalité d'un enfant qui, en devenant parent impliquera à son tour de façon inconsciente son enfant qui lui aussi verra sa capacité de développement être affectée. Il en va parfois ainsi pour plusieurs générations ${ }^{3}$.

La perte aura été vécue de façon traumatisante soit à cause de la nature de l'événement lui-même, de ses consé- quences pour la vie familiale ou encore pour des raisons d'ordre subjectif, comme des sentiments de culpabilité ou d'injustice, qu'ils soient fondés ou non ${ }^{4}$. L'impossibilité d'enclencher ou de mener à terme le processus de deuil installe dans le fonctionnement psychique individuel, comme dans les interactions familiales, des mécanismes d'adaptation puisant dans les réactions normales à une perte mais laissant par leur intensité et leur persistance des empreintes qui pourront être repérées, entre autres, dans la dynamique suicidaire d'un descendant.

Dans cette perspective, la problématique du suicide peut souvent être comprise comme une impasse dans la capacité d'individuation d'une personne liée d'une façon rigide à au moins un de ses parents, parfois dans la fusion, parfois dans la violence, parfois dans l'inceste (qu'il soit agi ou non au plan sexuel). La diversité apparente de ces liens cache toujours un point commun: une angoisse de séparation à l'origine d'une relation qui, d'une façon ou d'une autre, doit toujours rester soudée pour empêcher la réactivation de sentiments reliés à des pertes dans le passé du parent et de sa 
famille. Ce type de lien est souvent masqué ou compensé par des attitudes d'apparence opposée, ce qui peut en compliquer l'identification.

\section{LA REPRÉSENTATION GRAPHIQUE DES RÉPÉTITIONS}

Le génogramme a été développé à partir des idées de Murray Bowen ${ }^{5}$, psychiatre et thérapeute familial. Inspiré de l'arbre généalogique, il permet la représentation graphique de l'histoire d'une famille sur au moins trois générations, à partir de l'inscription des événements principaux (mariage, naissances, maladies, décès, etc.) jusqu'à certains faits de la petite histoire (départ de la maison, fin des études, rupture amoureuse, etc.) auxquels on ajoute tout ce qui est connu ou pensé des rapports entre les membres de la famille ainsi que certains traits psychologiques révélateurs ${ }^{6}$.

En plus de rendre présente et accessible pour le thérapeute comme pour l'individu et sa famille une quantité considérable d'informations, le génogramme permet le repérage essentiel de répétitions d'une génération à l'autre, de certains événements, comportements, modèles de rapports conjugaux, parentaux, à l'intérieur des fratries comme entre les familles d'origine des conjoints ${ }^{7}$. Bien que ses principes d'application peuvent varier selon les approches théoriques auxquelles se réfère le thérapeute, il permet habituellement de faire ressortir les principales problématiques vécues par les familles tout comme leurs forces et ressources pour y faire face ${ }^{8}$.

La prise de conscience de ces répétitions intergénérationnelles ${ }^{9}$, qu'elle soit faite par l'individu seul ou en présence d'autres membres de sa famille, a habituellement un impact significatif sur le degré de tension intérieure et relationnelle. Dans les situations de crise suicidaire, l'élaboration du génogramme peut agir sur le sentiment fréquent d'isolement et de perte d'appartenance psychique en remettant symboliquement la personne à sa place dans sa famille et dans sa lignée 10 . En donnant un sens au comportement problématique d'un parent ou d'un conjoint comme témoignant d'un héritage psychologique lié à un traumatisme ayant marqué la ou les générations antérieures, les apports cognitifs permettent une relativisation du problème et de son impact émotionnel. L'impossibilité de comprendre donne toujours plus d'emprise au désespoir et, à l'inverse quand la situation difficile devient perçue comme une conséquence plausible d'un enchaînement de faits (dont le comportement problématique du parent n'est qu'un des maillons), il devient alors possible de travailler à en maîtriser l'influence psychologique.

Pour notre part, nous utilisons le génogramme à partir d'un modèle de compréhension et d'intervention relié à l'approche familiale intergénérationnel$1 \mathrm{e}^{11}$, particulièrement autour des travaux de Murray Bowen (la différenciation de soi) et de Yvan Boszormenyi-Nagy («les loyautés invisibles», la thérapie contextuelle) ${ }^{12}$. Nous nous référons aussi aux travaux de certains psychanalystes français pour la compréhension des influences transgénérationnelles en jeu dans la problématique du suicide ${ }^{13}$ ainsi qu'à certains concepts issus de l'analyse systémique des interactions familiales ${ }^{14}$.

Exemple: (certains éléments biographiques ont été modifiés et le génogramme simplifié pour protéger l'anonymat de la famille)

Roger, dans la soixantaine, consulte pour une réaction dépressive à la suite du suicide de son fils aîné. Alain s'est jeté sur l'autoroute du haut d'un viaduc et s'est fait heurter mortellement par une voiture. Il s'est donné la mort peu après avoir appris que sa femme le trompait avec son frère plus jeune (Alexandre). Aux funérailles, la conjointe d'Alain serait sortie de l'église aux bras d'Alexandre. On aurait entendu des commentaires comme: "On est entré pour un service mais on est sorti d'une noce!» Le client est confus, atterré et se sent coupable envers Alain avec qui la relation avait toujours été conflictuelle: "Il me ressemblait trop». Il a préféré son deuxième qui lui faisait penser à son demi-frère plus jeune, Albert, qui lui aussi avait été préféré par leur père. "Ça doit venir de mon côté, on doit avoir ça dans le sang, mon père aussi s'est suicidé il y a une vingtaine d'années!» Roger raconte que son père s'est suicidé juste avant de venir habiter avec lui et sa famille.

Je propose au client d'explorer l'histoire de sa famille pour trouver un sens à ces événements tragiques. Il accepte ma suggestion d'inviter Albert, seul autre membre de sa génération encore vivant.

L'élaboration du génogramme familial leur a permis de reconnaître le sens de ces deux suicides en lien direct avec un enchaînement complexe d'événements traumatisants ayant profondément marqué leur enfance à tous les deux.

Roger a été le troisième et dernier enfant du premier mariage de leur père (Roméo). Il a perdu sa mère (Marthe) à
4 ans, décédée d'un cancer à l'âge de 38 ans. Leur père s'est remarié à peine six mois après le décès, avec la sœur cadette de sa première femme (Louisa). Roger et Albert ont tous deux entendu des rumeurs concernant le début d'une relation amoureuse avant le décès de la sœur aînée. Albert, seul enfant du second mariage, a vu sa mère devenir invalide après avoir été frappée par une voiture au cours d'une promenade en compagnie de son mari. Le jeune enfant âgé de deux ans était dans les bras de son père qui a réussi à se dérober à l'impact de la voiture. La fille aînée du premier mariage a dû remplacer sa tante (Louisa) pour les tâches domestiques et le soin des plus jeunes. Mais celle-ci est éventuellement décédée au même âge que sa sœur aînée et du même type de cancer. Albert et son père sont demeurés seuls durant près de dix ans après le départ des autres enfants, le fils devant assumer seul les tâches ménagères. Plutôt soumis et s'exprimant peu les conflits entre les deux étaient rares. Mais les choses ont changé après qu'Albert se soit marié: chicanes continuelles entre son père et sa femme (Louise), qui après la naissance de leur premier enfant, réclama ouvertement le départ de son beau-père. C'est dans ce contexte qu'il demande à son fils aîné, Roger, la permission d'aller habiter chez lui. Il lui offre de payer une pension mais en escomptant qu'Albert la paierait. C'est suite au refus catégorique d'Albert de payer la pension que leur père s'est pendu dans sa chambre.

Après la mort de sa mère, Roger avait grandi dans le rôle de bouc émissaire pour sa tante qui le critiquait constamment, le rendant responsable de ses migraines et autres douleurs physiques. Roger s'est rappelé avoir souvent espéré que son père prenne sa défense mais il est toujours demeuré distant et plutôt froid avec lui après s'être remarié. Roger a aussi vu son père préférer ouvertement son plus jeune fils. Il avait donc accepté avec joie la demande de son père d'habiter chez lui, espérant ainsi retrouver un peu de la proximité perdue suite à la mort de sa mère.

Le travail avec le génogramme et la remise en contexte de l'enchaînement des événements tragiques ayant marqué leur enfance jusqu'au suicide de leur père et celui d'Alain a d'abord permis d'assouplir un peu des sentiments de culpabilité extrêmement intenses ${ }^{15}$. Roger et Albert ont réalisé tous les deux qu'ils avaient porté chacun à sa façon une identification à leur mère respective décédée. Cette identification avait 


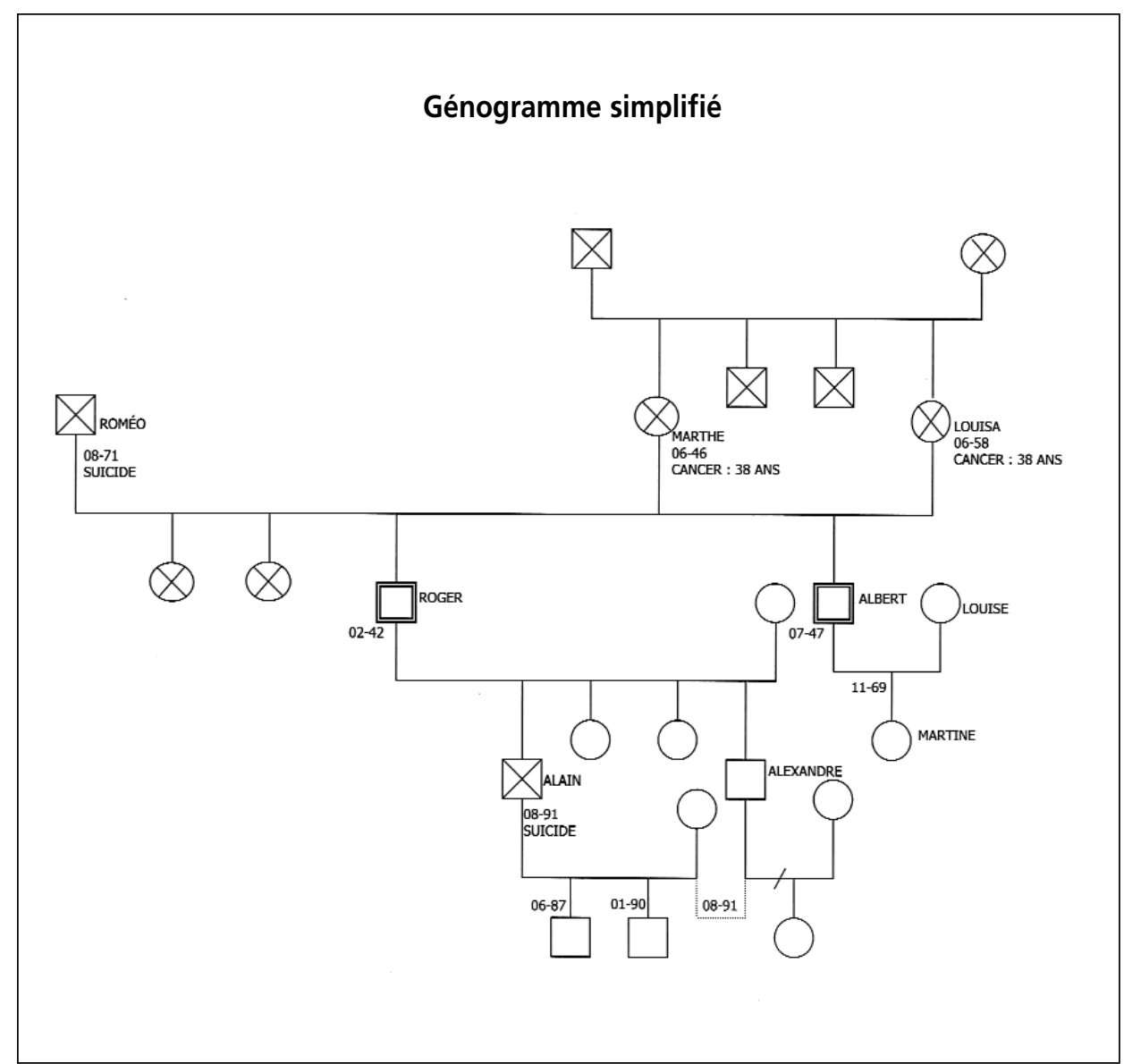

probablement permis la continuité de la vie familiale durement mise à l'épreuve de façon répétée ${ }^{16}$. Du côté de Roger, en devenant le bouc émissaire de sa tante qui dirigeait vers lui une partie des sentiments ambivalents à l'endroit de sa sœur aînée, perçue de façon irrationnelle comme s'étant vengée à travers l'accident de voiture. (On peut aussi poser l'hypothèse que la mort au même âge, de la même maladie, comporte des éléments d'identification à la sœur décédée et témoigne d'un processus de deuil bloqué ${ }^{17}$.) De la même façon que Roger avait eu à subir la perte du lien avec son père tel qu'il était avant la mort de sa mère, Albert avait eu à vivre à deux ans, suite à l'accident, un premier deuil de sa mère désormais incapable de prendre soin de lui et psychologiquement très changée. Durant son enfance, Albert est devenu pour toute la famille le porteur de la présence de sa mère telle qu'elle était avant l'accident. Moins besoin de faire face à cet autre traumatisme, elle était là telle qu'elle était,...en lui! À douze ans, quand elle est morte, cette identification a été accentuée sous la pression inconsciente du père qui lui a demandé de continuer à remplacer symboliquement sa mère durant toute son adolescence. dizaine d'années. Albert dit qu'il ne s'est jamais senti capable d'intervenir devant l'agressivité de Martine envers sa mère même quand les affrontements verbaux allaient jusqu'aux souhaits de mort. Peu de temps après le suicide de son beaupère, Louise a développé un problème de douleurs chroniques (ayant toujours résisté aux nombreuses consultations médicales et psychiatriques) qui l'a éventuellement rendue incapable de faire les tâches ménagères. À cause du refus de sa fille de participer aux travaux de la maison, Albert s'est vu obligé de reprendre en partie le rôle qu'il avait tenu auprès de son père durant son adolescence.

Parallèlement, Roger a pu situer le suicide de son fils dans la suite de ses propres souffrances d'enfant. Lui-même porteur de la présence de sa mère décédée dans la famille devant l'agressivité irrationnelle de sa tante à son endroit et le retrait affectif de son père, il a «encrypté» en lui ces événements selon les modes de pensée et le vécu affectif d'un enfant de quatre, cinq ans, période marquée d'emblée par les fantasmes de rivalité oedipienne pensés et ressentis comme pouvant causer la mort.

Ses deux fils ont donc puisé dans l'inconscient de leur père les matériaux identificatoires qui les ont menés jusqu'à la répétition tragique de ce scénario où un aîné meurt dans le contexte d'une relation amoureuse de sa conjointe avec son frère cadet. Autre élément de répétition: Alain a provoqué sa propre mort en se faisant écraser par une voiture comme la deuxième femme de son grand-père.

L'élaboration du génogramme a permis à Albert et Roger de se remémorer ensemble un passé où la réalité fort complexe de leurs deuils d'enfant a été submergée et assujettie aux demandes inconscientes des parents de les supporter dans leurs deuils difficiles à eux. Leur disponibilité psychique d'enfant a été mobilisée à travers des mécanismes identificatoires et interactionnels (identification à la personne décédée, rôle de bouc émissaire, retrait affectif, etc.) pour permettre la continuité de la vie de la famille. La prise de conscience de ce rôle familial, coûteux pour leur développement et encore très lourd à porter dans leur vie actuelle, a représenté pour eux l'élément clé du processus thérapeutique. Des entrevues conjugales et familiales ont fait suite pour compléter la démarche entreprise par les deux demifrères. L'état de santé de Louise s'est éventuellement amélioré, alors que Martine a entrepris une psychothérapie 
individuelle après avoir traversé une période dépressive avec idéations suicidaires, suite à l'amélioration de la communication entre son père et sa mère. Le pouvoir excessif que lui avait conféré son rôle dans l'équilibre fragile de sa famille faisait place à un profond sentiment d'impuissance face à sa vie. Les repères identificatoires liés à l'appartenance à son sexe et à sa génération avaient été balayés au profit de cette forte identification à son grand-père. Le développement de sa personnalité en avait été fortement influencé.

La reconnaissance d'une telle contribution à l'équilibre familial est souvent au cœur de l'intervention en contexte de risque suicidaire, particulièrement à l'adolescence où le conflit entre devenir soi-même et demeurer fidèle à son rôle familial est parfois perçu comme sans issue. Même quand la dynamique est moins évidente, l'adolescent suicidaire porte souvent une peur secrète que son mouvement d'individuation et de séparation ne porte atteinte à la survie d'un parent ou du couple parental tout en étant habituellement ambivalent et incertain dans son désir d'autonomie. L'intervention intergénérationnelle favorise la mise à jour de ces enjeux familiaux en évitant le blâme et la culpabilisation, condition essentielle pour un dénouement positif de la crise.

\section{MISES EN LIEN ET QUÊTES D'ÉLABORATION}

Les prises de conscience, ouvertures émotionnelles et déblocages relationnels, qui accompagnent habituellement l'élaboration du génogramme, ne remplacent pas un travail de psychothérapie s'il est nécessaire. Mais, l'occasion, il semble que certaines personnes réussissent avec ces mises en lien à retrouver une certaine maîtrise de leur état intérieur et à agir sur leur situation. Il n'est pas rare, par ailleurs, que l'intervention familiale démontre un besoin de travail thérapeutique pour un autre membre de la famille que celui ou celle qui portait le risque suicidaire.

En l'absence de collaboration de la famille, l'élaboration du génogramme peut malgré tout favoriser chez la personne suicidaire un regard différent sur le sens et l'origine de son désespoir. Quand la collaboration est possible, elle permet d'établir rapidement l'implication et l'alliance thérapeutique avec l'ensemble des membres de la famille ${ }^{18}$. Par la quête d'information et l'intérêt pour les différents points de vue concernant le problème, le thérapeute se démarque d'emblée des polarisations et des conflits. Les questions concernant les naissances, décès, mariages, séparations, etc. décentrent l'attention de la situation de crise, qui n'est bien souvent que le reflet indirect des vraies blessures jamais guéries et reliées aux grands événements de l'histoire familiale. L'élaboration du génogramme donne ainsi un recadrage rapide de la crise suicidaire en donnant vite l'accès aux enjeux familiaux fondamentaux ${ }^{19}$.

La dimension concrète de la tâche elle-même mais aussi l'organisation des informations dans leur disposition graphique, semble souvent favoriser une certaine réorganisation du climat émotionnel débordé par l'intensité des sentiments soulevés en situation de crise.

Les résistances d'un membre de la famille ou d'une famille entière au travail avec le génogramme sont souvent révélatrices de loyautés rigides envers les générations précédentes, liées à des situations marquées par la honte ou la culpabilité (suicide, maladie mentale, inceste, naissance hors mariage, etc.). Silences, dénis, secrets installent dans la vie familiale et entre les générations une communication codée (mythes familiaux, rituels, modes relationnels) qui contient toujours des éléments révélateurs des traumatismes d'origine. Cette communication qui maintient aussi l'injonction de continuer à les taire. Avec le passage des générations, des familles peuvent donc vivre des violences ou des silences reliés à des événements dont elles n'ont aucune idée et qui pourraient être vécus comme des banalités dans la vie d'aujourd'hui ${ }^{20}$.

L'ensemble des questions concernant les événements importants, mais aussi la petite histoire comme les études, les déplacements géographiques, les héritages, les traits de ressemblance avec un parent, contacts avec les grands-parents, etc., sont toutes de nature à ramener à la conscience familiale plein de vécus relationnels conflictuels (injustices, rivalités, etc.) jamais réglés dans le contexte où ils se sont déroulés. Ce déballage de la petite histoire contribue aussi à «désengorger» l'accumulation des héritages ayant traversé les générations et dont la personne suicidaire porte habituellement plus que sa part.

Il est aussi parfois assez étonnant de voir comment la représentation graphique des personnes décédées, remises à leur place au sein de leur fratrie, leur couple, etc., ravive la mémoire des faits mais parfois aussi celle des émotions ayant entouré les pertes. L'évocation des ascendants dont le deuil n'a pu être fait est particulièrement impressionnante quant à l'occasion qu'elle donne de remettre à l'ordre du jour un travail de deuil impossible dans le contexte où était survenue la perte. C'est donc à travers ses manifestations transgénérationnelles (dont la crise suicidaire est un élément majeur) que ce travail de deuil s'élabore, par des allers-retours entre le scénario original (compris dans son contexte social, historique, culturel) et sa remise en scène dans la vie actuelle de la famille 21 .

Un tel travail serait bien sûr inaccessible à l'individu ou à la famille sans les appuis cognitifs qu'offre le génogramme par la mise à jour d'éléments de répétition, marqueurs de l'itinéraire des séquelles de traumatismes toujours en quête d'élaboration (coïncidences de dates, répétition de prénoms, de faits, etc.).

L'objectif principal de l'utilisation du génogramme est de briser l'isolement psychique et relationnel de la personne menaçant de se suicider (ou «menacée» de suicide) et de permettre à tous les membres de la famille de récupérer leur part du problème. Il est évident que ce travail d'intervention familiale présuppose que l'urgence suicidaire est contenue, que la personne à risque est en sécurité et qu'il y a un minimum de collaboration dans la famille. Cependant, bien des familles seront accessibles à un travail de psychothérapie seulement dans le contexte de l'imminence d'une tentative, au moment de l'hospitalisation ou juste après. Il faut donc prévoir différents modèles d'intervention selon le contexte et les diverses dynamiques familiales et être prêt à intervenir rapidement, particulièrement avec les systèmes familiaux très rigides.

Le génogramme est tout autant un outil d'évaluation que d'intervention. Son utilisation en contexte de risque suicidaire doit cependant être envisagée avec prudence et exige une bonne expérience de travail clinique. Rien à voir avec l'enquête ou la chasse au secret. L'essentiel du processus est de favoriser une reprise du dialogue et une remise en lien. Nous avons rencontré à quelques occasions des situations où la rigidité des attitudes et des loyautés à l'égard des ascendants semblait l'emporter sur le désir d'éviter le sacrifice d'un membre de la famille, comme si la survie psychique du groupe familial le réclamait. Des réserves doivent aussi être émises concernant l'utilisation du génogramme avec des familles dont la structure fragile pourrait être débordée par l'intensité émotionnelle soulevée par cette démarche. Par ailleurs, l'abord des causalités psychiques transgénérationnelles 


\section{Éléments relationnels}

Descriptions d'éléments relationnels :

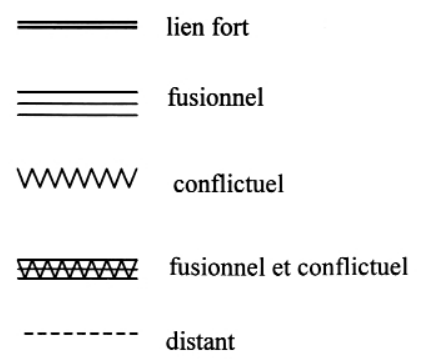

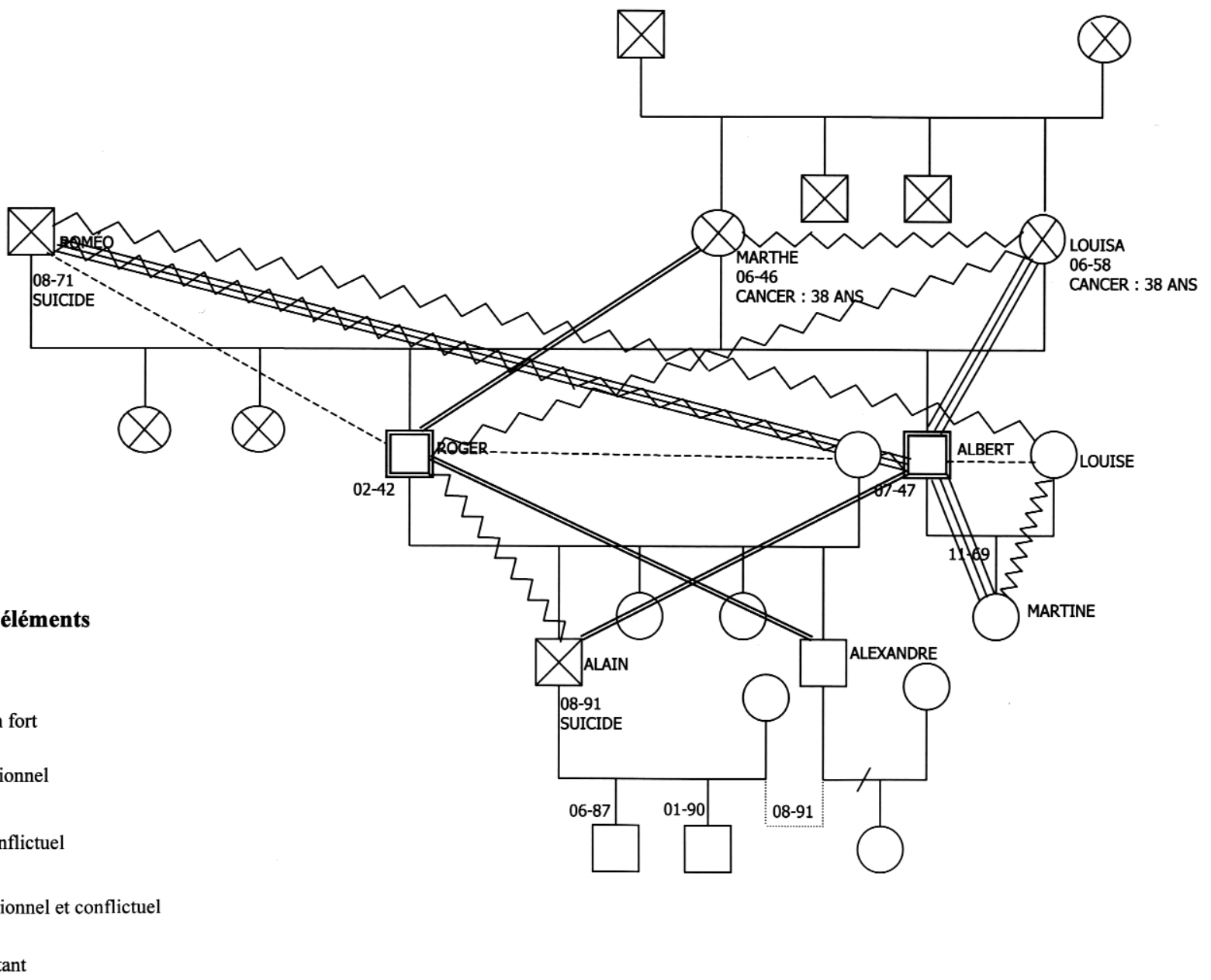

pourrait s'avérer particulièrement indiqué dans l'intervention auprès des familles marquées par la répétition de pertes par suicide avec la possibilité de facteurs génétiques dans l'étiologie des passages à l'acte ${ }^{22}$.

Le fait de responsabiliser l'individu suicidaire face à sa vie et de responsabiliser, sans les culpabiliser, les membres de sa famille représente à notre point de vue une des mises à l'épreuve les plus fortes dans le travail de psychothérapie. Ce défi est certainement facilité par une bonne connaissance de soi: compréhension de son rôle dans sa propre histoire familiale et de sa motivation profonde à exercer ce métier. La prise de conscience de la force méconnue de certains héritages psychologiques peut nous choquer à cause de l'aspect implacable de certains déterminismes, comme elle peut mener vers un sentiment de liberté reconquise par leur mise à jour. Nos propres «loyautés invisibles» peuvent provoquer l'impression de lever le voile sur un savoir tabou, dont l'accès doit nous demeurer interdit en rapport avec l'image intériorisée de nos liens familiaux. Ce type d'intervention implique aussi une bonne capacité de dialogue et de résolution de conflits à l'intérieur des équipes d'intervention où se déplacent trop souvent les polarisations portées par les familles en souffrance.

La mise à jour des influences inter et transgénérationnelles s'avère souvent utile et parfois décisive dans la clinique $\mathrm{du}$ risque suicidaire. Comme pour bien d'autres problématiques à causalité multifactorielle (problèmes de développement ou comportement chez les enfants et les adolescents, problèmes conjugaux, somatisation, toxicomanie, anorexie-boulimie, etc.) leur importance relative peut varier. Toutefois, la compréhension des enjeux familiaux approfondit et facilite habituellement les interventions, peu importe l'approche ou la technique utilisée.

Accompagner les familles dans cette démarche généalogique éveille beaucoup de curiosité pour mieux connaître l'environnement socioculturel où sont survenus les pertes et traumatismes non surmontés. Il semble bien que les processus de deuil bloqués et la transmission de leurs conséquences se déroulent souvent dans un contexte d'asymétrie de pouvoir ${ }^{23}$, que ce soit à l'intérieur des familles comme au plan social: pouvoir des adultes sur les enfants, des hommes sur les femmes, d'une idéologie religieuse rigide sur une population captive, domination culturelle ou politique.

Il n'est pas rare d'entendre une grand-mère pleurer et parler pour la première fois devant sa famille de la perte d'un enfant en cours de grossesse ou mort-né; perte parfois associée à un contexte de violence conjugale ou encore à une directive d'oublier vite et d'en fabriquer un autre pour que le berceau ne soit pas vide lors de la prochaine visite paroissiale, sinon... Au cours d'une intervention familiale faisant suite à une tentative de suicide d'un de ses petit-fils, une de ces grand-mères a l'occasion de réactiver son travail de deuil en présence de ses enfants et petits-enfants qui en 
portaient les traces. L'élaboration du génogramme a fait ressortir que le père de l'adolescent suicidaire avait vite remplacé un petit frère décédé dans le berceau. Il avait aussi hérité du même prénom... Son fils ne s'est jamais senti reconnu et aimé de lui. Il lui reproche sa froideur et son intransigeance. Mais ce père «absent» a fondu en larmes en voyant son fils consoler sa grand-mère quand elle évoque ce petit bébé dont elle n'a jamais fait le deuil et dont lui-même a partagé le berceau, le prénom et porté la présence pour sa mère endeuillée, ayant essayé lui aussi, à sa façon et dès le début de sa vie, de la consoler...

Révoltes et sentiments d'injustice jamais exprimés sont intériorisés et engendrent leur lot de deuils impossibles. Les liens familiaux deviennent surchargés de la présence des morts qui symboliquement réclament toujours reconnaissance et parfois réparation. Cette «solidarité» avec les morts se transmet cependant à même un cycle de destruction et d'autodestruction qui hypothèque les rapports entre générations au plan familial comme au plan collectif. Par exemple, le discours des Premières Nations sur les problèmes sociaux et la prévalence du suicide dans certaines de leurs communautés en témoigne éloquemment ${ }^{24}$. Certains de nos deuils collectifs attendraient-ils encore d'être sortis du déni ${ }^{25}$ ?

\section{Notes}

1 J. RICHMAN, Family Therapy for Suicidal People, New York, Springer, 1986.

2 F. WALSH et M. McGOLDRICK, Living beyond Loss, Death in the Family, NewYork, W.W. Norton and Company, 1991.

3 S. TISSERON et al., Le psychisme à l'épreuve des générations, Paris, Dunod, 1995.

C. NACHIN, Les fantômes de l'âme, Paris, L'Harmattan, 1993.

4 C. FIGLEY, The Traumatology of Giving. Death and Trauma, Washington, DC, Taylor and Francis, 1997.

5 M. BOWEN, La différenciation du soi, Paris, E.S.F., 1984.

6 M. MC GOLDRICK et R. GERSON, Génogramme et entretien familial, Paris, E.S.F., 1990.

7 A. ANCELIN-SCHÜTZENBERGER, Ä̈e mes aïeux!, Paris, Épi La Méridienne, 1993.

8 E. LEMAIRE-ARNAUD, «Le génogramme en thérapie conjugale et familiale», Dialogue, 1995.

9 E. LEMAIRE-ARNAUD, «Utilité du génogramme pour la mise à jour des phénomènes transgénérationnels", Dialogue, no 89,1985 , p. 3-7.

R. BREUER, «Les failles de la symbolisation familiale et leur repérage, étude clinique», Dialogue, AFCCC, no 4, 1996, p. 7-16.

10 J.L. FRAMO, Family of Origin Thérapy, N.X., Brunner-Mazel, 1992.

11 G. SALEM, «Approche intergénérationnelle et problèmes de setting en thérapie familiale», Thérapie familiale, vol. 9, no 2, 1988, p. 135-145.

12 Y. BOSZORMENYI-NAGY et J.L. FRAMO, Psychotherapies Familiales, Paris, P.U.F., 1980.

Y. BOSZORMENYI-NAGY et G. SPARK, Invisible Loyalties: Reciprocity Intergenerational Family Therapy, Hagerstown, Harper et Row, 1973.

G. SALEM, "Boszormenyi-Nagy et le grand livre des dettes et mérites», Dialogue, AFCCC, no 4, 1990, p. 50-70.

13 N. ABRAHAM et M. TOROK, L'écorce et le Noyau, Paris, Aubier, 1978.

R. KAËS, Transmission de la vie psychique entre générations, Paris, Dunod, 1993.

A. EIGUER, et al., Le générationnel, Paris, Dunod, 1997.

D. DUMAS, Hantise et clinique de l'Autre, Paris, Aubier, 1989.

14 D. VALLÉE, «Pour une suicidologie systémique», Thérapie familiale, Genève, vol. 9, no 2, 1988, p. 159-165.

D. SAMPAIO, «Tentative de suicide dans l'adolescence: une perspective systémique», Thérapie familiale, Genève no 3, 1985, p. 281-291.

C.R., PFEFFER, «The Family System of Suicidal Children», American Journal of Psychotherapy, no 35, 1981, p. 303-341.

15 J. COURNUT, «Deuil et sentiment de culpabilité dans «Le Deuil»», Revue Française de Psychanalyse, Paris, P.U.F., 1994.
M. HANUS, «Le Travail de deuil, dans «Le Deuil»», Revue Française de Psychanalyse, Paris, P.U.F., 1994

16 M. POROT, L'enfant de remplacement, Paris, Éd. Sciences et culture, 1994.

17 P.-C. RACAMIER, Le génie des origines, Paris, Payot, 1992

18 M. CANNELS, "Le génogramme et son processus», Dialogue, AFCCC, no 4 1994, p. 53-63.

19 G. SALEM, L'approche thérapeuthique de la famille, Paris, Masson, 1987.

20 E. GRANJON, «Traces et effets de traumatismes psychiques transgénérationnels», Revue de psychothérapie psychanalytique de groupe, no 24, 1996, p. 65-71.

21 N. D'ARAGON, L'Approche familiale intergénérationnelle dans l'intervention auprès des personnes suicidaires et leur famille, Association Québécoise de suicidologie, Actes du Colloque, 1998.

22 J.-L. PEDINIELLI, "Par où passent les secrets de famille: Suicide et généalogie: la filiation suicidante», Dialogue, AFCCC, no 4, 1994, p. 38-50.

23 E.R. SHAPIRO, Grief as Family Process, London, Guilford Press, 1996.

24 M.C. SINCLAIR, «Suicide in First Nations Peolple», Suicide in Canada, Toronto, University of Toronto Press, 1998.

25 P. CADY «Métissés serrés. Surgissement de l'originaire d'un peuple dans sa littérature: l'exemple québécois», Filigrane, vol. 6, no 2, 1997.

D. DELAGE, Le pays renversé, Montréal, Boréal, 1991. 